\title{
INFRARED EMISSION AT HIGH GALACTIC LATITUDE
}

\author{
F. Boulanger \\ Ecole Normale Supérieure \\ 24 rue Lhomond \\ 75005 Paris, France
}

\begin{abstract}
Results obtained with the Infrared Astronomy Satellite (IRAS) on the IR emission at high galactic latitude are reviewed. We present evidence for the detection of galactic emission at $12,25,60$, and $100 \mu \mathrm{m}$. We describe the morphology of this emission and summarize work on the correlation of IR cirrus with $\mathrm{H} \mathrm{I}, \mathrm{CO}$, and optical emission. We discuss the contribution of the neutral atomic, ionized, and molecular gas to the total IR emission and the contribution of different components of interstellar dust to the emission seen at different wavelengths.
\end{abstract}

\section{INTRODUCTION}

The all-sky survey carried out by the Infrared Astronomy Satellite (IRAS) at 12, 25, 60, and $100 \mu \mathrm{m}$ provided the first observations of the IR emission from interstellar matter at high galactic latitude. At $100 \mu \mathrm{m}$ the sensitivity of the survey corresponded to the emission from a column density of matter of a few $10^{19} \mathrm{H} \mathrm{cm}^{-2}$. With this sensitivity IRAS detected emission from the Galaxy over most of the sky. These observations have been used to probe and compare the spatial distribution of dust and gas, to study the size distribution of interstellar dust grains, and to discuss the origin of the IR emission of the Galaxy. Work on these data is reviewed in this paper with the intention of providing a general description of the main features of the high galactic latitude sky in the IR. In section 2 we present evidence for the existence of a galactic background at the four wavelengths of the IRAS survey and discuss its origin. The galactic emission has a complex morphology with filamentary structures named IR cirrus after their resemblance to clouds in the terrestrial atmosphere. We discuss the association of IR cirrus with atomic and molecular interstellar clouds, optical nebulosity, and visual extinction (section 3). The correlation between the IR and H I emission seems to indicate that the IR emission arises mostly from dust associated with neutral atomic gas, although one-fourth of the interstellar gas at high galactic latitude is ionized. The question of the contribution of the ionized component of the interstellar medium (ISM) is addressed in section 4. Minima of IR emission at high galactic latitude allow regions of little extinction and diffuse emission to be selected. These are the best windows for extragalactic studies. We analyze the emission in these regions in section 5 .

\section{GALACTIC BACKGROUND}

Figure 1 presents galactic latitude profiles of the IR emission obtained by Boulanger and Pérault (1988, hereafter BP) after subtraction of zodiacal light. Data in the direction of the Magellanic Clouds, the main nearby molecular clouds and OB associations, and a few bright point sources were discarded when computing these profiles. An H I profile built over the 


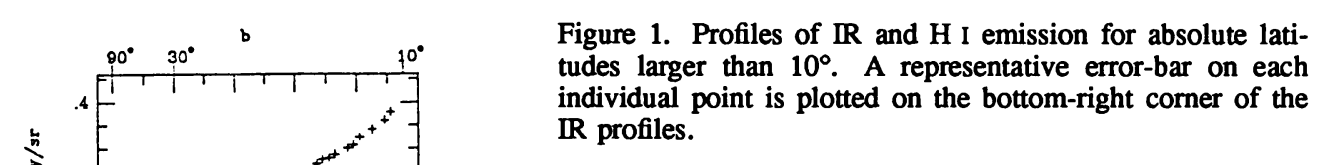

same parts of the sky as the IR profiles is also presented in Figure 1. The fact that the latitude profiles follow a cosecant law proves the existence of galactic emission for all $b$ at all IRAS wavelengths. The subtraction of the zodiacal light is particularly critical for the detection of the galactic emission at 12 and $25 \mu \mathrm{m}$, which at $|b|>10^{\circ}$ represents less than $1 \%$ of the zodiacal light in the ecliptic plane. The absence of emission above $30^{\circ}$ in the 12 and $25 \mu \mathrm{m}$ profiles results directly from the procedure used by $\mathrm{BP}$ to model the zodiacal light and does not mean that there is no galactic emission at 12 and $25 \mu \mathrm{m}$ all the way to the poles. From the slopes of the cosecant laws, BP derived the average spectrum of the IR emission at high galactic latitude listed in Table 1 . This spectrum is extended to the near-IR by the $3.3 \mu \mathrm{m}$ balloon measurement of Giard et al. (1989) at a few degrees above the molecular ring and to the submillimeter waveband by the rocket measurements reported by Lange et al. (1989).

The question of the origin of the IR emission measured by the cosecant laws can be addressed by comparing its spatial structure about the average latitude profile with the distribution of interstellar matter. BP computed for each $0.5^{\circ} \times 0.5^{\circ}$ pixel all-sky maps of $60 \mu \mathrm{m}, 100 \mu \mathrm{m}$, and $\mathrm{H} \mathrm{I}$ emission the difference between the observed emission and the average brightness at the galactic latitude of the pixel. A pixel-bypixel comparison of these differences is presented in Figure 2. This figure shows a clear correlation between the structure in the IR and $H$ I maps. The straight lines on the $60 \mu \mathrm{m}-\mathrm{H}$ I and $100 \mu \mathrm{m}-\mathrm{H}$ I

diagrams represent the IR emissivity per $\mathrm{H}$ atom derived from the cosecant laws (see Table 1 ). The fact that these lines approximately follow the major axis of the distribution of points in the IR-H I diagrams implies that the far-IR emission at high galactic latitude arises mainly from dust associated with neutral atomic gas or from some other gas component whose spatial distribution is correlated with that of H I. Uncertainties in the zodiacal light subtraction are too large to allow a pixel-by-pixel correlation between the 12 and $25 \mu \mathrm{m}$ emission and the distribution of interstellar dust over the whole sky. However, one can indirectly prove that the 12 and $25 \mu \mathrm{m}$ emission comes from the ISM by showing that stars and circumstellar dust-shells account for only a small fraction of the observed emission. On the basis of the IRAS catalog and the deep IRAS survey near the north ecliptic pole $\left(b=30^{\circ}\right)$ analyzed by Hacking and Houck (1987), BP estimated that photospheres and circumstellar dust-shells account for about $10 \%$ of the $12 \mu \mathrm{m}$ and $2.5 \%$ of the $25 \mu \mathrm{m}$ emission seen at $|b|>10^{\circ}$. Therefore, most of 
the galactic emission at 12 and $25 \mu \mathrm{m}$ comes from the ISM.

TABLE 1. Energy Distribution of IR Emission at High Galactic Latitude

\begin{tabular}{cc}
\hline \hline$\lambda$ & $\begin{array}{c}4 \pi v I_{\mathrm{v}} \\
\left(10^{-31} \mathrm{~W} / \mathrm{H}\right)\end{array}$ \\
\hline $3.3 \mu \mathrm{m}^{a}$ & $1.9 \pm 0.4$ \\
$12 \mu \mathrm{m}$ & $1.1 \pm 0.3$ \\
$25 \mu \mathrm{m}$ & $0.7 \pm 0.2$ \\
$60 \mu \mathrm{m}$ & $1.1 \pm 0.06$ \\
$100 \mu \mathrm{m}$ & $3.2 \pm 0.12$ \\
$137 \mu \mathrm{m}$ & $5.0 \pm 1.0^{b}$ \\
$262 \mu \mathrm{m}$ & $0.9 \pm 0.4^{b}$
\end{tabular}

${ }^{a}$ Intensity at the peak of the $3.3 \mu \mathrm{m}$ feature from Giard et al. (1989); FWHM is $0.05 \mu \mathrm{m}$; total intensity in the feature is $2.9 \times 10^{-33} \mathrm{~W} / \mathrm{H}$; level of the continuum emission around the feature is unknown. ${ }^{b}$ Spectrum from Lange et al. (1989) scaled to the $100 \mu \mathrm{m}$ flux from IRAS.
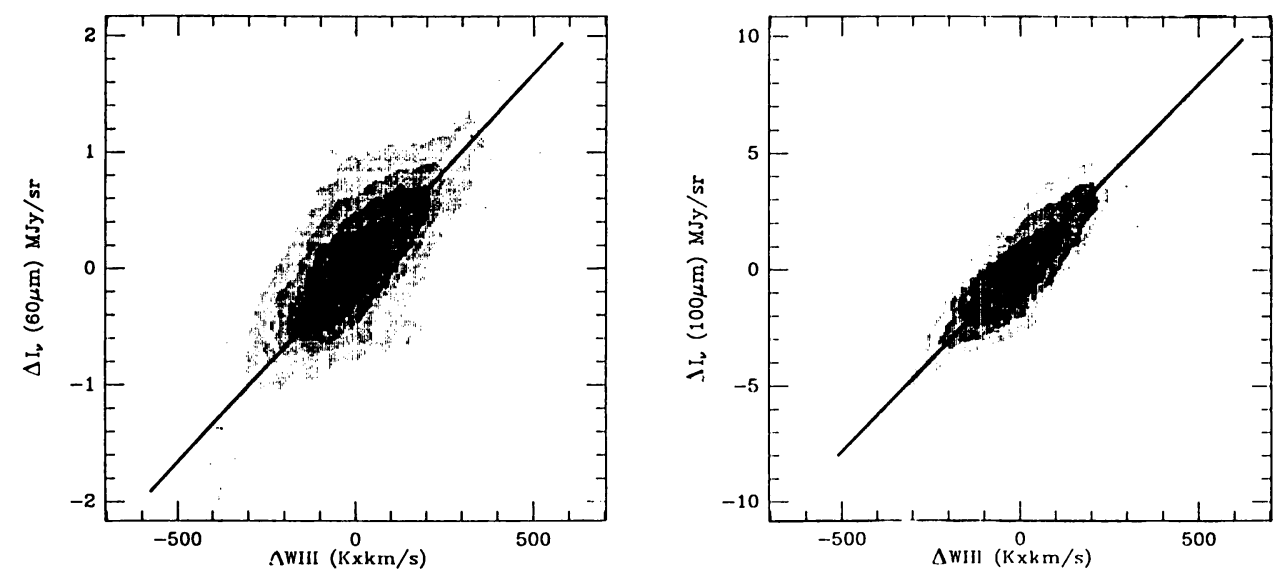

Figure 2. Correlation between IR and $\mathrm{H} \mathrm{I}$ emission at $|b|>10^{\circ}$. For each pixel in the $60 \mu \mathrm{m}, 100$ $\mu \mathrm{m}$, and $\mathrm{H} \mathrm{I}$ all-sky maps, we computed the difference between the map value and the average emission at the galactic latitude of the pixel. The two gray-scale plots show the density distribution of these differences in a $60 \mu \mathrm{m} \mathrm{m}-\mathrm{H}$ I and a $100 \mu \mathrm{m}-\mathrm{H}$ I diagram. The straight lines superposed on the grayscale plots go through the origin and have slopes equal with the ratios of 60 and $100 \mu \mathrm{m}$ to $\mathrm{H}$ I emission derived from the cosecant laws. Pixels in the Magellanic Clouds and in the vicinity of the main nearby molecular clouds and $\mathrm{OB}$ associations were not included in these plots.

In the spectrum listed in Table 1, one sees that the power radiated by interstellar dust is distributed over a wide range of wavelength from the near-IR to the submillimeter. Since dust at high latitude is illuminated by a rather homogeneous radiation field, the emission must originate from different types of particles emitting at different temperatures. The IRAS data, therefore, confirmed the discovery, first made in reflection nebulae (Sellgren, Werner, and Dinerstein 1983), of the existence in the ISM of small particles transiently heated to high temperatures by the absorption of single photons. Those results not accounted for by dust models 
proposed only on the basis of extinction and scattering data (Mathis, Rumpl, and Nordsieck 1977; Draine and Lee 1984) force us to change our view of interstellar dust. In an attempt to reproduce the spectrum in Table 1 at the same time as the extinction curve, Désert, Boulanger, and Puget (1989) concluded that there must be at least three different dust components, two of which are small particles emitting through temperature fluctuations: (1) polycyclic aromatic hydrocarbons molecules (PAHs), made of about 50 to 200 carbon atoms, which account for the near-IR emission and part of the mid-IR, (2) very small grains (VSGs), three-dimensional particles with sizes between 1.5 and $5 \mathrm{~nm}$, contributing to the 25 and $60 \mu \mathrm{m}$ emission measured by IRAS, and (3) large dust grains in thermal equilibrium with the radiation field emitting in the far-IR and the submillimeter. PAHs and VSGs each account for $\sim 20 \%$ of the total emission, large grains for the remaining $60 \%$.

From the emissivities per H-atom listed in Table 1, Désert et al. (1990) estimated the bolometric emission of interstellar matter in the IR and submillimeter to $7 \times 10^{-31} \mathrm{~W} / \mathrm{H}$. Only half of this emission is radiated within the range of wavelengths $(8-120 \mu \mathrm{m})$ covered by IRAS. Dispersion measurements in the direction of pulsars indicate that about $25 \%$ of the interstellar matter at high galactic latitude is ionized (Reynolds 1989). If the diffuse ionized gas seen at high galactic latitude has an IR emissivity similar to that for neutral atomic gas (see section 4), the IR emission per nucleon is $5 \times 10^{-31} \mathrm{~W} / \mathrm{H}$. This IR emissivity should be equal to the amount of stellar light absorbed by dust, which may be estimated from the interstellar radiation field, the extinction curve, and albedo measurements. For the values tabulated by Mathis, Mezger, and Panagia (1983) and the standard $A_{v} / N_{\mathrm{H}}$ ratio, the power absorbed by dust is $4.3 \times 10^{-31} \mathrm{~W} / \mathrm{H}$, which is in reasonable agreement with the IR emissivity if we take into account existing uncertainties of dust albedos. The slight difference may indicate that albedos are smaller than those obtained by Mathis, Rumpl, and Nordsieck (1977) with their silicate plus graphite dust model.

\section{INFRARED CIRRUS}

In the first series of papers published on IRAS observations, Low et al. (1984) reported the discovery of highly structured extended sources of far-IR emission, which they describe as "IR cirrus." In this early report Low et al. gave several examples of IR cirrus which coincided spatially with $\mathrm{H}$ I clouds but which also pointed out that many extended structures did not have any clear counterpart in H I maps. Since this paper, much work has been done in associating IR cirrus with interstellar clouds seen in $\mathrm{H} \mathrm{I}$ or $\mathrm{CO}$, or as nebulosity or extinction patches in optical plates.

Boulanger, Baud, and van Albadal (1985), Terebey and Fich (1986), de Vries, Heithausen, and Thaddeus (1987), and BP have investigated the correlation between far-IR and $\mathrm{H}$ I data over several regions of the sky, typically a few tenths of a degree in size. Within each of the fields analyzed by these authors there is a tight correlation between the far-IR and $\mathrm{H}$ I emission wherever there are no molecular clouds. As an example we present in Figure 3 a pixel-by-pixel comparison between the $100 \mu \mathrm{m}$ and $\mathrm{H} \mathrm{I}$ emission in the northern and southern polar cap. The good correlation observed within each of the fields that was analyzed proves that, on scales of the order of $100 \mathrm{pc}$, the interstellar radiation field and the dust abundance are reasonably uniform. Figure 2, which gathers data over the whole sky at $|b|>10^{\circ}$ away from OB associations and the Magellanic Clouds, provides a general impression of the IR-H I correlation. In these diagrams the far-IR and $\mathrm{H}$ I emission appear clearly correlated, but the scatter of the data, points around the correlation line is $\sim 2$ times larger than what is expected from the 
uncertainties on the infrared and $\mathrm{H}$ I data, indicating that there is not a simple one-to-one correspondence between the far-IR and $\mathrm{H}$ I maps.
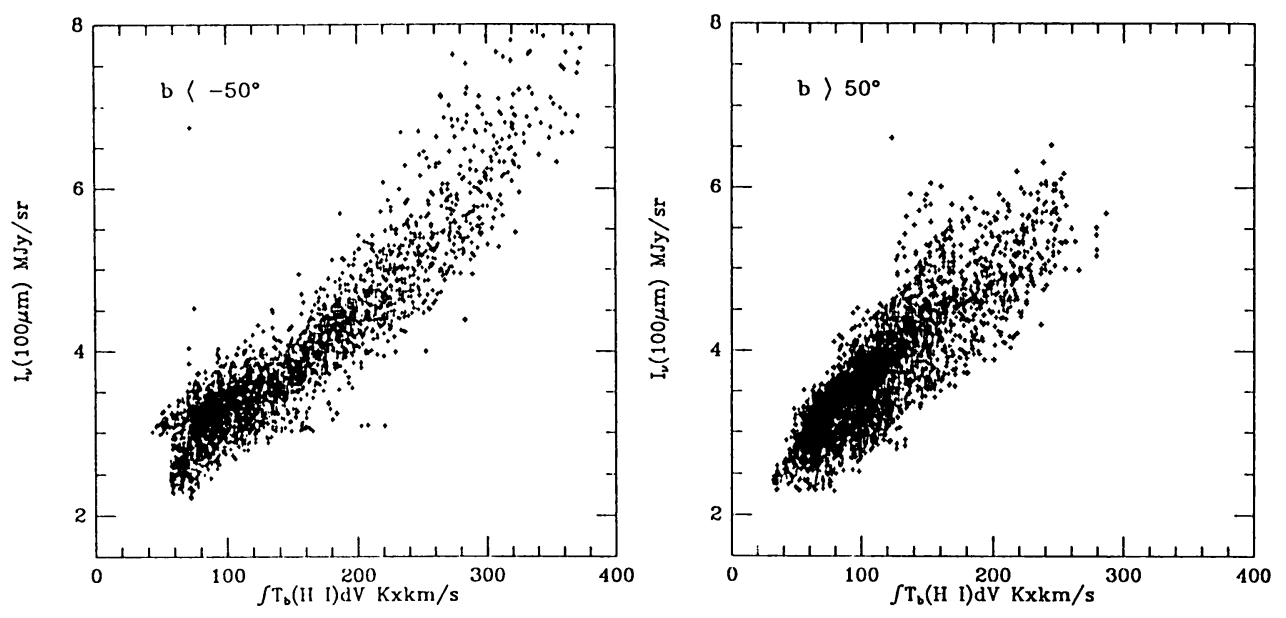

Figure 3. Pixel-by-pixel comparison between $100 \mu \mathrm{m}$ and $\mathrm{H} \mathrm{I}$ emission in the northern and southern polar caps. To avoid contamination by stray radiation, we used for this comparison the $\mathrm{H}$ I survey made with the horn reflector of the Bell Laboratories. The $100 \mu \mathrm{m}$ data were smoothed to the lower resolution of this survey; each pixel represents a $1.5^{\circ} \times 1.5^{\circ}$ pixel.

Part of the scatter in the diagrams of Figure 2 results from variations in the IR emission per $\mathrm{H}$ atom from place to place in the solar neighborhood. A systematic analysis of the 100 $\mu \mathrm{m}-\mathrm{H}$ I correlation was carried out by Désert, Bazell, and Boulanger (1988, hereafter DBB), who built a map of the $100 \mu \mathrm{m}$ emissivity per $\mathrm{H}$ atom from the results of comparisons within cells of $20^{\circ}$ in longitude and $8^{\circ}$ in latitude. At $|b|>20^{\circ}$, away from the Sco-Cen and Orion OB associations, the $I_{\mathrm{v}}(100 \mu \mathrm{m}) / N_{\mathrm{H}}$ ratio is rather uniform and remains within $\sim 50 \%$ of the cosecant law value $0.86 \mathrm{MJy} \mathrm{sr}^{-1}$ for $10^{20} \mathrm{H} \mathrm{cm}^{-2}$. More important variations are observed at lower latitudes and close to $\mathrm{OB}$ associations. At low latitude in the direction of the outer parts of the Galaxy, the $I_{\mathrm{v}}(100 \mu \mathrm{m}) / N_{\mathrm{H}}$ ratio is about a factor 2 lower than the cosecant value (Terebey and Fich 1986). In the Lupus region, close to the Sco-Cen OB association, BP measured a ratio 3 times higher than the cosecant value. The fact that high values of the far-IR emissivity are observed in the vicinity of $O B$ associations, while low values are observed towards the external parts of the Galaxy, suggests that changes in the IR emission per $\mathrm{H}$ atom result from variations in the intensity of the interstellar radiation field rather than from changes in the dust-to-gas ratio.

Scatter in Figure 3 also results from the presence of molecular clouds at high galactic latitudes. The launch of the IRAS satellite and the observation of IR cirrus coincided with the discovery of small molecular clouds at high galactic latitude by Blitz, Magnani, and Mundy (1984) and Magnani, Blitz, and Mundy (1985). Weiland et al. (1986) and de Vries, Heithausen, and Thaddeus (1987) were the first ones to associate several high latitude molecular clouds with IR cirrus. They found a ratio between $I_{\mathrm{v}}(100 \mu \mathrm{m})$ and the $\mathrm{CO}$ emission integrated in velocity WCO of $1.0 \pm 0.5\left(\mathrm{MJy} \mathrm{sr}^{-1}\right) /\left(\mathrm{Kkm} \mathrm{s}^{-1}\right)$. From this ratio and the survey of Magnani, Blitz, and Mundy (1985), BP estimated that the small molecular clouds observed at high latitude account for $<1 \%$ of the total $100 \mu \mathrm{m}$ emission over the same latitude range, 
which is not surprising since these clouds account for a small fraction of the gas surface density: $\sigma\left(H_{2)} \sim 0.1 M_{\odot} \mathrm{pc}^{-2}\right.$ while $\sigma(\mathrm{H} \mathrm{I}+\mathrm{H} \mathrm{II}) \sim 6 M_{\odot} \mathrm{pc}^{-2}$. Although high latitude clouds seen in $\mathrm{CO}$ account for a negligible fraction of the far-IR emission, numerous cirrus clouds or structure within the cirrus do not have an $\mathrm{H}$ I counterpart. The systematic comparison of 100 $\mu \mathrm{m}$ and $\mathrm{H}$ I data of DBB was carried out with the purpose of establishing a catalog of regions with an excess IR emission from what is expected from gas seen in emission in $\mathrm{H} \mathrm{I}$. This analysis led to a list of 578 clouds with excess IR emission distributed over the whole sky. The resolution of the DBB analysis was limited to $0.5^{\circ}$. This resolution precludes them from detecting excess-IR regions with extents smaller than about a degree. As a result, some cloudlets detected in CO by Magnani, Blitz, and Mundy do not appear in their list. DBB suggested that most of these clouds correspond to high latitude molecular clouds. However, Désert et al. (1990) surveyed these clouds for CO emission and detected only approximately one-third of the clouds. Clouds not detected in $\mathrm{CO}$ could be molecular but without $\mathrm{CO}$ (see Lada and Blitz 1988) or made of cold neutral atomic gas. One surprising result of the analysis of DBB is the existence of a number of regions with a deficit of IR emission, compared with the emission expected from $\mathrm{HI}$ gas, as large as the number of excess clouds. There is no obvious correlation between these deficit regions and the presence of $\mathrm{H}$ I gas at intermediate velocities. Globally, regions with excess or deficit IR emission with respect to the IR-H I correlation represent only a small fraction, $\sim 2 \%$, of the total IR emission.

The $10^{\circ} \times 12^{\circ} \mathrm{H} \alpha$ map centered at $l=144^{\circ}, b=-21^{\circ}$, presented by Reynolds (1980) indicates that the diffuse ionized gas has a complex morphology made of sharp filaments and some more diffuse regions. Features of ionized gas in these maps correspond to column densities of $-5 \times 10^{19} \mathrm{~cm}^{-2}$, which should be detectable in the IRAS data if they have an IR emissivity similar to that of $\mathrm{H}$ I clouds. However, no attempt to look for the IR counterparts of these $H \alpha$ structures has been reported.

The presence of interstellar dust at high galactic latitude was inferred from nebulosity in Schmidt plates many years before the IRAS launch (see, e.g., de Vaucouleurs and Freeman 1972; Sandage 1976). Shortly after the report of the discovery of IR cirrus, de Vries and Le Poole (1985) looked for the optical counterpart of two cirrus clouds and showed that they could be seen as regions of faint diffuse emission in the blue plates of the ESO/SERC sky survey. For a peak $100 \mu \mathrm{m}$ brightness between 5 and $15 \mathrm{MJy} \mathrm{sr}^{-1}$, these clouds had a maximum optical brightness between 24 and $25 \mathrm{mag} \operatorname{arcsec}^{-2}$. From this analysis de Vries and Le Poole concluded that with a detection limit of 27 mag $\operatorname{arcsec}^{-2}$, the southern optical survey has a sensitivity to high latitude cirrus similar to that of IRAS. Laureijs, Mattila, and Schnur (1987) did a similar comparison on $\mathrm{L} 1642$ for several wavelengths from the $\mathrm{U}$ to the $\mathrm{V}$ band and were able to model successfully the optical emission as galactic light scattered by dust in the cloud. On the basis of data over a wider range of wavelength obtained by Mattila (1979), Chlewicki and Laureijs (1987) pointed out that in the red the brightness of the high latitude cloud L1780 is much larger than what is expected from scattered light, and they suggested that the excess emission is fluorescence from PAHs. A much more detailed study of the optical emission of high latitude cirrus has been recently carried out by Guhathakurta and Tyson (1989) employing a mosaic of deep CCD frames. Their observations generalize to clouds of smaller optical depth the existence of an excess of emission in the $R$ and $I$ band above what is expected from scattered light. For one cloud the blue emission is an order of magnitude smaller than what is expected from isotropic scattering. For the other clouds, like for the results of de Vries and Le Poole (1985) and Laureijs, Mattila, and Schnur (1987), the ratio of blue to $100 \mu \mathrm{m}$ emission, $I_{\mathrm{v}}(0.44 \mu \mathrm{m}) / I_{\mathrm{v}}(100 \mu \mathrm{m})$, is in close agreement with the value of $\sim 3 \times 10^{-3}$ expected from scattering. 
Comparison of the $100 \mu \mathrm{m}$ IRAS data with $\mathrm{H}$ I and CO data show that the $100 \mu \mathrm{m}$ brightness, away from local heating sources, is well correlated with the distribution of atomic and molecular gas. For molecular clouds a good correlation is also observed between the 100 $\mu \mathrm{m}$ brightness and the visual extinction derived from star counts with slopes between 5 and 10 (MJy sr${ }^{-1}$ ) $\mathrm{mag}^{-1}$ (de Vries and Le Poole 1985; and review by Boulanger 1989). The good correlation between $100 \mu \mathrm{m}$ emission and gas and dust column density indicates that the large dust grains at the origin of the $100 \mu \mathrm{m}$ emission are well mixed with the gas. Emission at shorter wavelengths is not as well correlated with the gas distribution. Numerous studies of the IR emission of interstellar clouds heated by the average interstellar radiation field of the Galaxy (Boulanger, Baud, and van Albada 1986; Leene 1986; de Vries and Le Poole 1985; Weiland et al. 1986; Heiles, Reach, and Koo 1988; Laureijs, Mattila, and Schnur 1987; Laureijs, Chlewicki, and Clark 1988) have shown than the ratio between the mid-IR (12 and 25 $\mu \mathrm{m}$ ) and the $100 \mu \mathrm{m}$ emission varies by more than one order of magnitude from cloud to cloud. In a recent study, Boulanger et al. (1990) have shown evidence for color variations of similar amplitude on scales as small as $-0.5 \mathrm{pc}$ within the nearby molecular complexes of Taurus and Chamaeleon and within the high latitude clouds in Ursa Major. Boulanger et al. (1990) have argued that color variations result from variations in the abundance of PAHs and VSGs (section 2) and not from an excitation effect due to variations in the UV radiation field as proposed by Chlewicki and Laureijs (1988) and Beichman et al. (1988). Heiles, Reach, and Koo tried to relate color variations with gas velocity but found only marginal evidence for any correlation. Small particles have absorption and scattering properties different from those of large dust grains. Changes in dust composition indicated by color variations must therefore be related to the variations observed in the UV part of the extinction curve (Massa and Savage 1989), although there is presently little observational evidence.

IRAS images of high latitude cirrus have been used to study the power spectrum of high latitude cirrus (Gautier and Boulanger 1989). The shapes of the power spectra were found to be well represented by a power law. Two-dimensional analyses yield indices near -3 from $0.125 \mathrm{deg}^{-1}$ to the Nyquist limit of the data $0.5 \operatorname{arcmin}^{-1}$. The spectral index was generally found to be substantially independent of azimuth in the frequency space. The power spectrum analysis made on images was extended to lower frequencies by one-dimensional analyses, which yielded spectral indices near -2 down to frequencies as low as $0.07 \mathrm{deg}^{-1}$. The difference of about unity between the one- and two-dimensional analyses results from the dimension change in the Fourier transform. The spectral indices found for IR cirrus are similar to those obtained by Crovisier and Dickey (1983) from single velocity maps of the $\mathrm{H} \mathrm{I}$ emission in the Galaxy.

\section{CONTRIBUTION FROM DIFFUSE IONIZED GAS}

The interstellar matter seen at $|b|>20^{\circ}$ is mostly atomic gas, about three-fourths of it neutral and one-fourth ionized (see Reynolds 1989). Unless there is a difference in dust abundance between neutral and ionized gas, the ionized component of the nearby ISM must account for $-25 \%$ of the IR emission measured by the cosecant laws. However, if we scale the latitude distribution of $\mathrm{H}$ I emission by the $I_{\mathrm{v}}(100 \mu \mathrm{m}) / N_{\mathrm{H}}$ ratio derived from the scattered diagrams in Figure 3, we get a latitude distribution which differs from the profile of IR emission by much less than the $25 \%$ contribution expected from diffuse ionized gas (see Figure 4). Note that the $I_{\mathrm{v}}(100 \mu \mathrm{m}) / N_{\mathrm{H}}$ ratio derived from the diagrams in Figure 3 measures the IR emissivity of individual H I clouds because there is hardly any variation of the background emission with 
galactic latitude at $|b|>50^{\circ}$. Since the $\mathrm{HI}$ and $100 \mu \mathrm{m}$ profiles in Figure 4 agree closely at all latitudes, this problem cannot be solved by simply changing the zero level of the galactic emission (see section 5) because the column density of ionized gas increases with the cosecant of the latitude (Reynolds 1984). The close agreement seen between the two profiles in Figure 4 could be coincidental if the IR emissivity of the $\mathrm{H}$ I gas were decreasing with latitude just to
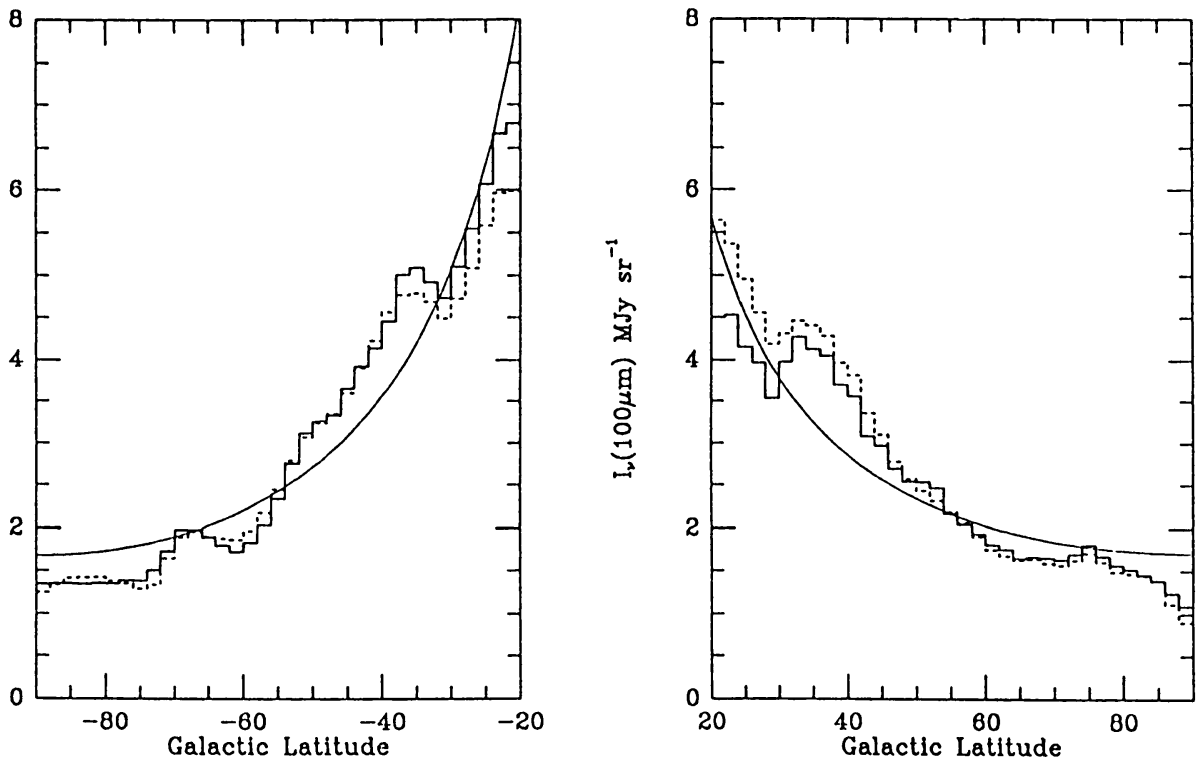

Figure 4. Galactic latitude profiles of the $100 \mu \mathrm{m}$ emission at $b<-20^{\circ}$ and $b>20^{\circ}$. In each plot the histogram drawn with a solid line represents the $100 \mu \mathrm{m}$ profile. The dotted histogram represents the profile of the integrated $\mathrm{H}$ I emission derived from the survey of the Bell Laboratories and scaled by the $I_{\mathrm{v}}(100 \mu \mathrm{m}) / \mathrm{W} \mathrm{H}$ I ratios derived from the $100 \mu \mathrm{m}-\mathrm{H}$ I correlation at $b>50^{\circ}$ and $b<-50^{\circ}$ (Figure 3 ). For the southern profiles, only the part of the sky covered by the Bell Laboratories survey was used. In each plot the continuous line represents a least-squares fit of the $100 \mu \mathrm{m}$ profile by a cosecant law plus a constant. These cosecant laws are explicitly formulated in BP.

compensate for the increase of the emission of the ionized gas. But analyses of the 100 $\mu \mathrm{m}-\mathrm{H}$ I correlation (BP, DBB) show on the contrary that $I_{\mathrm{v}}(100 \mu \mathrm{m}) / N_{\mathrm{H}}$ increases slightly from the polar caps to $|b|=20^{\circ}$. Therefore, we see only two possible explanations to the coincidence of the two profiles in Figure 4: (1) the distribution of ionized gas is correlated with the distribution of the $\mathrm{HI}$ gas, or (2) the diffuse ionized gas is deficient in dust. In the first case the $100 \mu \mathrm{m}$ emissivities derived from the slope of the $100 \mu \mathrm{m}-\mathrm{H}$ I correlation will include the contribution of ionized gas. Some degree of correlation between the spatial distribution of neutral and ionized gas is expected in the three-phase picture of the ISM where ionized gas is found in transition regions between $\mathrm{H} \mathrm{I}$ clouds and hot coronal gas (McKee and Ostriker 1977). Reynolds (1980) and Munch (1989, this volume) have pointed out that some of the structures seen in $\mathrm{H} \alpha$ maps appear spatially and kinematically correlated with $\mathrm{H}$ I filaments and clouds, but not all of the diffuse ionized gas can be at the surface of $\mathrm{H}$ I clouds since the scale height of the ionized gas is much greater than that of neutral clouds (Lockman 1984; Reynolds 1989). Larger maps are necessary to clarify the degree of correlation between $\mathrm{H} \alpha$ and $\mathrm{H} \mathrm{I}$ 
emission. Because of a lower density, the rate of destruction of grains by interstellar shocks is much higher in the warm phase than in the cold phase of the ISM (McKee et al. 1987). If the cycling time of the gas between these two phases is larger than the time scale for dust destruction in the warm phase, $-3 \times 10^{7} \mathrm{yr}$, this phase could be significantly depleted in dust compared with the cold phase, which could be an explanation for proposition (2) above.

\section{EXTRAGALACTIC WINDOWS}

One of the motivations in analyzing the galactic emission at high latitude is the desire to be able to model and subtract it from the total emission in order to discuss the existence of an extragalactic background. Gray-scale plots of the distribution of the $100 \mu \mathrm{m}$ emission around the northern and southern galactic poles down to $|b|=30^{\circ}$ have been presented by BP. Regions of low-IR emission can be identified on these plots. These regions are the best windows for extragalactic studies. The regions of low $\mathrm{H}$ I column density investigated by Lockman, Jahoda, and McCammon (1986) are among these windows. In these regions the $100 \mu \mathrm{m}$ emission associated with neutral atomic gas is $\sim 1 \mathrm{MJy} \mathrm{sr}^{-1}$. This corresponds to a column density of hydrogen of $\sim 10^{20} \mathrm{H} \mathrm{cm}^{-2}$ and a visual extinction $A_{v} \sim 0.05$. There is possibly some ionized gas in these holes of IR emission which could produce some additional extinction and IR emission. If there is as much ionized gas in the holes as on average at high latitude, for an emissivity similar to that for the $\mathrm{H} \mathrm{I}$ gas, the $100 \mu \mathrm{m}$ emission from ionized gas would be $-0.8 \mathrm{MJy} \mathrm{sr}^{-1}$. After subtraction of the contribution from $\mathrm{H}$ I gas, the $100 \mu \mathrm{m}$ brightness in the holes of IR emission is $1.8 \pm 0.3 \mathrm{MJy} \mathrm{sr}^{-1}$. The error bar on this number does not include uncertainties in the zero-level calibration of the IRAS data, or the potential contribution from ionized gas. Evidence for this unaccounted brightness comes directly from the $100 \mu \mathrm{m}-\mathrm{H}$ I diagrams of Figure 3, where correlation lines have clear non-zero intercepts. A detailed analysis of the absolute calibration is currently in progress (Gautier et al. 1990) to ascertain the significance of this unaccounted brightness.

Acknowledgments-I am grateful to Drs. R. Harmon and J. L. Puget for a careful reading of the manuscript.

\section{REFERENCES}

Beichman, C., et al. 1988, Ap. J., 332, L81.

Blitz, L., Magnani, L., and Mundy, L. 1984, Ap. J. (Letters), 282, L9.

Boulanger, F., Baud, B., and van Albada, G. D. 1985, Astr. Ap., 144, L9.

Boulanger, F., and Pérault, M. 1988, Ap. J., 330, 964 (BP).

Boulanger, F. 1989, The Physics and Chemistry of Interstellar Molecular Clouds, eds. G. Winnewisser and J. T. Armstrong (Springer Verlag), in press.

Boulanger, F., et al. 1989, Ap. J., submitted.

Chlewicki, G., and Laureijs, R. J. 1987, Polycyclic Aromatic Hydrocarbons and Astrophysics, eds. A. Léger, L. d'Hendecourt, and N. Bocarra (Reidel), p. 335.

Chlewicki, G., and Laureijs, R. J. 1988, Astr. Ap., 207, L11.

Crovisier, J., and Dickey, J. M. 1983 Astr. Ap., 122, 282.

Désert, F. X., Bazell, D., and Boulanger, F. 1988, Ap. J., 334, 815 (DBB).

Désert, F. X., Boulanger, F., and Puget, J. L. 1989, Astr. Ap., submitted.

Désert, F. X., et al. 1989, in preparation.

Draine, B. T., and Lee, H. M. 1984, Ap. J., 285, 89.

de Vaucouleurs, G., and Freeman, K. C. 1972, Vistas Astron., 14, 163.

de Vries, C. P., and Le Poole, R. S. 1985, Astr. Ap., 145, L7. 
de Vries, H. W., Heithausen, A., and Thaddeus, P. 1987, Ap. J., 319, 723.

Gautier, T. N. G., and Boulanger, F. 1989, in preparation.

Gautier, T. N. G., et al. 1990, in preparation.

Giard, M., et al. 1989, Astr. Ap., 215, 92.

Guhathakurta, $\mathbf{P}$., and Tyson, J. A. 1989, Ap. J., in press.

Hacking, P., and Houck, J. R. 1987, Ap. J. Suppl., 63, 311.

Heiles, C., Reach, W. T., and Koo, B. C. 1988, Ap. J., 332, 313.

Lada, E., and Blitz, L. 1988, Ap. J., 326, L69.

Lange, A. E., et al. 1989, Interstellar Dust: IAU Symposium No 135, eds. L. Allamandola and A. G. G. M. Tielens (Reidel), in press.

Laureijs, R. J., Mattila, K., and Schnur, G. 1987, Astr. Ap., 184, 269.

Laureijs, R. J., Chlewicki, G., and Clark, F. O. 1988, Astr. Ap., 192, L13.

Leene, A. 1986, Astr. Ap., 154, 295.

Lockman, F. J. 1984, Ap. J., 283, 90.

Lockman, F. J., Jahoda, K., and McCammon, D. 1986, Ap. J., 302, 432.

Low, F. J., et al. 1984, Ap. J. (Letters), 278, L19.

Magnani, L., Blitz, L., and Mundy, L. 1985, Ap. J., 295, 402.

Massa, D., and Savage, B. D. 1989, Interstellar Dust: IAU Symposium No. 135, eds. L. Allamandola and A. G. G. M. Tielens (Reidel), in press.

Mathis, J. S., Rumpl, W., and Nordsieck, K. H. 1977, Ap. J., 217, 425.

Mathis, J. S., Mezger, P.G., and Panagia, N. 1983, Astr. Ap., 128, 212.

Mattila, K. 1979, Astr. Ap., 78, 253.

McKee, C. F., et al. 1987, Ap. J., 318, 674.

Munch, G. 1989, in Proc. IAU 139, Galactic and Extragalactic Background Radiation, ed. S. Bowyer and Ch. Leinert (Dordrecht: Kluwer Academic Publishers).

Reynolds, R. J. 1980, Ap. J., 236, 153.

Reynolds, R. J. 1984, Ap. J., 282, 191.

Reynolds, R. J. 1989, Ap. J., 339, L29.

Sandage, A. 1976, Ap. J., 81, 954.

Sellgren, K., Werner, M. W., and Dinerstein, H. L. 1983, Ap. J. (Letters), 271, L13.

Terebey, S., and Fich, M. 1986, Ap. J. (Letters), 309, L73.

Weiland, J. L., et al. 1986, Ap. J., 306, L101. 